\title{
Scanning and Transmission Electron Microscopy Studies of Alkali Halide Films Grown by Pulsed Laser Deposition
}

\author{
Dwight R. Acosta ${ }^{1}$, Elsi Mejía ${ }^{2}$, Citlali Sánchez ${ }^{2}$, Jimena Martinez $^{2}$, Carlos Magaña ${ }^{1}$ \\ 1. Instituto de Física \\ 2. Centro de Ciencias Aplicadas y Desarrollo Tecnológico \\ Universidad Nacional Autónoma de México
}

Pulsed laser deposition (PLD) technique has been used the last decades, to produce thin films of an ample diversity of materials. This technique uses high power laser pulses to melt, evaporate and ionize material from a target. The vaporized material consists of neutral atoms, ions, electrons, molecules, etc. which constitute a transient, highly luminous plasma plume that expands rapidly away from the target surface.

Film growth occurs on an appropriate placed substrate in which the ablated material is collected. The technique of PLD offers some advantages over other film deposition methods as the possibility to produce films of the exact chemical composition of the target and that relatively high deposition rates can be achieved. There are only few reports of laser ablation and PLD of alkali halides [1-2].There are a large number of variables affecting the properties of the plasma which in turn affect the characteristics of the deposited films, such as laser fluence, background gas pressure and substrate temperature. These parameters allow manipulating the films properties to suit particular applications. In this work we report the growth of $\mathrm{NaCl}$ and $\mathrm{KCl}$ films by PLD, using different laser fluences and distances between the target and the substrate.

Alkali halide films were deposited under ablation of commercial targets of $\mathrm{NaCl}$ and $\mathrm{KCl}$ with an excimer laser (COMPex 102, Lambda Physik), emitting at $248 \mathrm{~nm}$ and with a pulse duration of $30 \mathrm{~ns}$ (FWHM). Films were deposited at room temperature on 200 mesh copper TEM grids previously fixed on corning glass laminates and using 6,000 laser shots. The substrates were placed in front of the target at distances in the range from 4 to $10 \mathrm{~cm}$. Deposition was carried out in a stainlesssteel vacuum chamber evacuated until a base pressure of 1 x 10-6 Torr. The laser beam was focused using a planeconvex lens with focal length of $50 \mathrm{~cm}$, leading to fluences from 0.2 to $1 \mathrm{~J} / \mathrm{cm} 2$.

Alkali halides $(\mathrm{NaCl}$ and $\mathrm{KCl})$ have been succesfully deposite on carbon and resin substrates using the pulsed laser deposition technique for different deposition times and different substrates temperature. TEM, SEM and STEM micrographs reveal details of coalescense and growth processes at different stages :a) nanocrystallites and b) microcrystallites. The last ones reveal the growth in a self assemble way following crystallites faceting. It has been found that even for short deposition times the coalescence and growth occurs simultaneously at nano and micro size levels and the resulting alkali halide films are produced after a continuous coalescence and assembly processes.

References:

1. A. Arrieta, S. Mera, R. Diamant, M.Fernández, R.Sosa, L.Escobar, A. Muñoz, E. Haro; Appl. Physics S491-S493, 1999

2 .P.E.Mayer, S.M. Maswadi, C.D.Walton; Appl. Phys. A76, 817-822, 2 


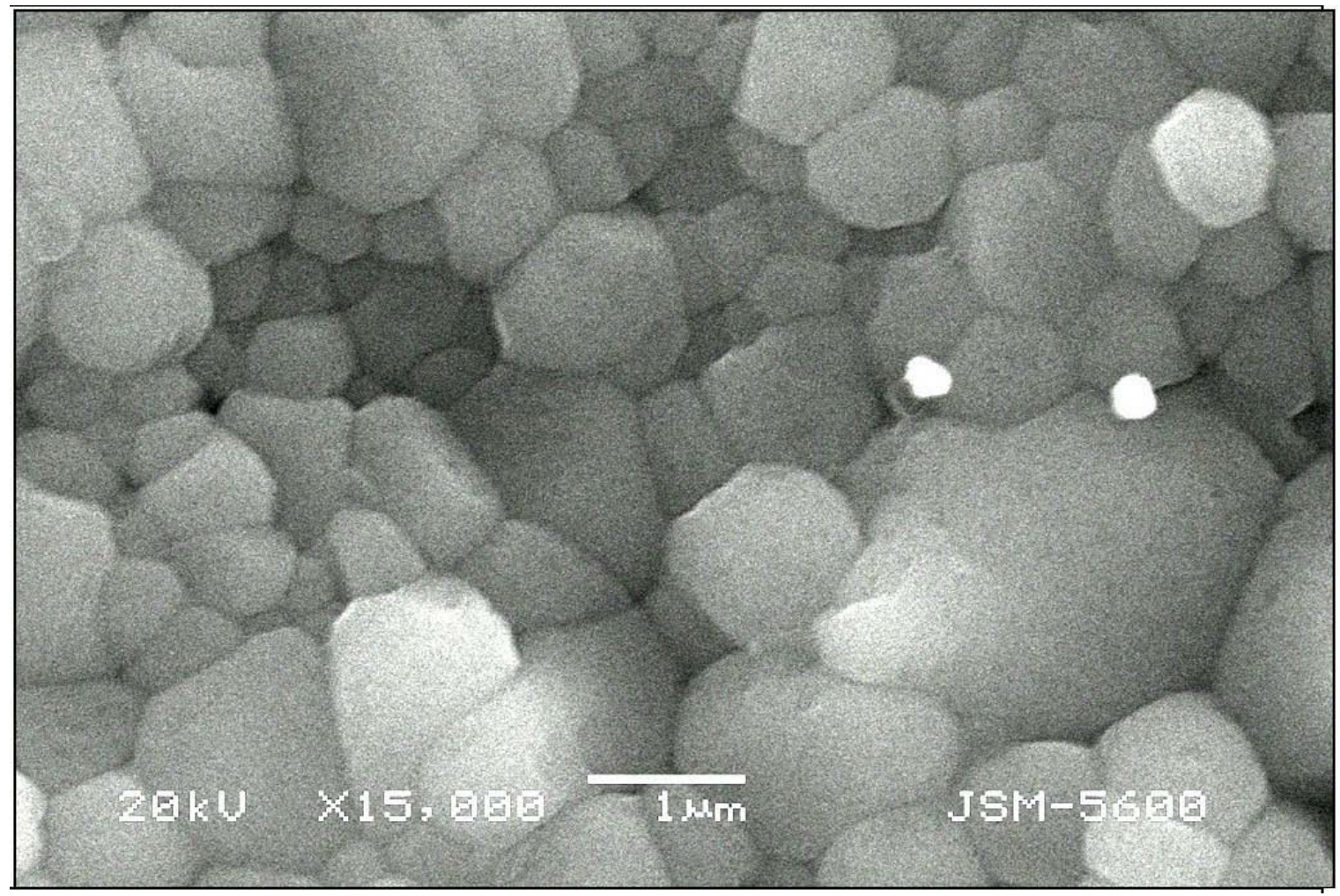

Figure 1. SEM micrograph of a $\mathrm{KCl}$ sample deposited on a substrate at $300^{\circ} \mathrm{C}$. The film surface looks dense, regular and compact.

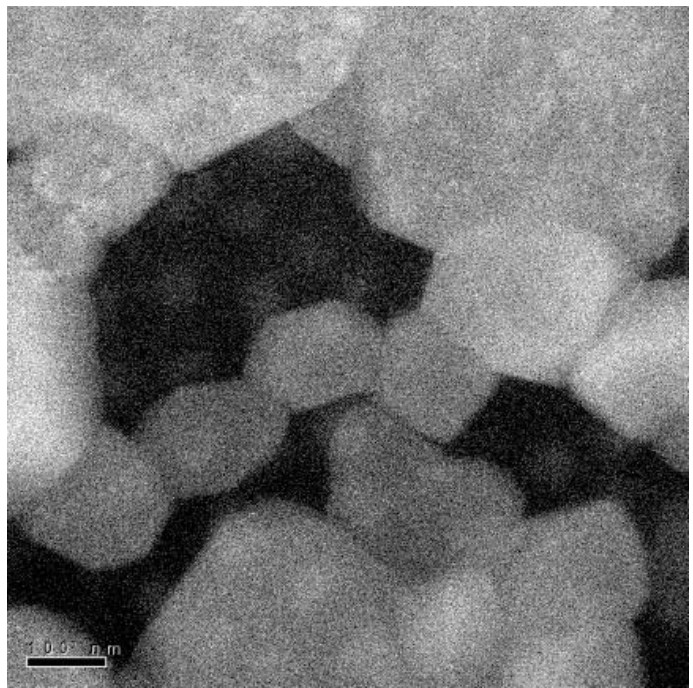

Figure 2. A DF STEM micrograph of $\mathrm{NaCl}$ displays the film growth when the micro-crystallites are self-assembled following specific crystallographic orientations.

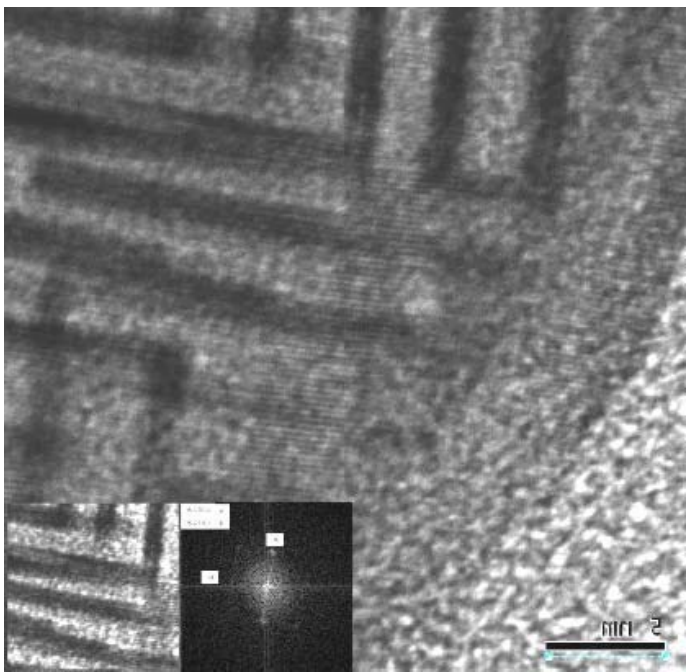

Figure 3. HREM micrograph of a PLD deposited $\mathrm{NaCl}$. The typical terrace configuration can be observed. Also the FFT and FFT.-1 of the central zone are displayed. 